\title{
1 Einleitung
}

Im Jahr 1979 gab Klaus Magyar, Stadtrat und Kreisarzt in Halle (Saale), die Broschüre Prophylaxe im industriellen Ballungsgebiet Halle heraus. ${ }^{9}$ Darin enthalten war ein Beitrag von Gerd Münx, Hartmut Bruns und Gottfried Rudolf mit dem Titel Venerische Erkrankungen und ihre Prophylaxe in der Stadt Halle. ${ }^{10}$ Die drei Autoren arbeiteten am Bezirkskrankenhaus Halle (Saale), der Poliklinik Mitte: Obermedizinalrat (OMR) Dr. med. Gerd Münx (1918-200o) war Facharzt für Haut- und Geschlechtskrankheiten, Dr. phil. Gottfried Rudolf war DiplomPsychologe in der Abteilung Dermato-Venerologie und Hartmut Bruns leitete als Medizinal-Assistent die Abteilung Statistik. ${ }^{11}$ Gleich zu Beginn des vierseitigen Artikels wurde von Münx und seinen Kollegen ein Krisenszenario entworfen: „Die Syphilis-Neuerkrankungen nehmen seit dem Nachkriegs-Tiefstand von 1970 langsam, aber beständig wieder zu und haben 1977 in der DDR

9 Magyar K (Hrsg.) (1979) Prophylaxe im industriellen Ballungsgebiet Halle. Beitrag des kommunalen Gesundheitswesens der Stadt Halle und der Martin-Luther-Universität Halle-Wittenberg zum 30. Jahrestag der Gründung der Deutschen Demokratischen Republik. Halle (Saale).

10 Münx G, Bruns H, Rudolf G (1979) Venerische Erkrankungen und ihre Prophylaxe in der Stadt Halle. In: Magyar $\mathrm{K}$ (Hrsg.) Prophylaxe im industriellen Ballungsgebiet Halle. Beitrag des kommunalen Gesundheitswesens der Stadt Halle und der Martin-Luther-Universität Halle-Wittenberg zum 30. Jahrestag der Gründung der Deutschen Demokratischen Republik. 69-72. Halle (Saale).

11 Autorenverzeichnis. In: Magyar K (Hrsg.) (1979) Prophylaxe im industriellen Ballungsgebiet Halle. Beitrag des kommunalen Gesundheitswesens der Stadt Halle und der Martin-Luther-Universität Halle-Wittenberg zum 30. Jahrestag der Gründung der Deutschen Demokratischen Republik. X-XIII. Halle (Saale). 
mit einer Zahl von 574 einen neuen Höhepunkt erreicht. Ähnlich, aber noch krasser, verhält er sich mit der Entwicklung der Neuerkrankungen an Gonorrhoe. (...) Das Jahr 1977 weist mit 45.209 Erkrankungen eine Erkrankungshäufigkeit auf, die der des Jahres 1947 bedenklich nahe kommt. " ${ }^{12}$ In der Lesart von Münx, Rudolf und Bruns befand sich die DDR im Jahr 1977 also im unmittelbaren Nachkriegszustand, wenn man die entsprechenden Statistiken über Geschlechtskrankheiten richtig las und interpretierte. Nach einer Reduktion der Infektionsraten wären die Raten seit Anfang der 197oer Jahre wieder rasant angestiegen. Dieses Krisenszenario gab aber nur einen Teil der Situation in der DDR wieder. Ein Blick in einzelne Bezirke und Bezirksstädte der DDR vervollständigte das Gesamtbild und ließ Differenzierungen zu. Dabei fiel Münx und seinen Kollegen vor allem die Situation in Halle (Saale) auf: „Halle liegt in den Erkrankungshäufigkeiten je 1.ooo der Wohnbevölkerung sowohl über dem DDR-als auch über dem Bezirksdurchschnitt. Auch die Anstiegsrate ist überdurchschnittlich hoch. " ${ }^{13}$ Zudem würden in Halle (Saale) zwei weitere Besonderheiten besonders deutlich zutage treten: „Der überdurchschnittliche Anstieg an Erkrankungen in Halle geht nämlich ausschließlich zu Lasten der Frauen. Während die Männer in Halle die dem Republik-Durchschnitt entsprechende Zuwachsrate von 9\% aufweisen, ist bei den Frauen ein Zuwachs von $42 \% \mathrm{zu}$ verzeichnen. " ${ }^{14}$ Und nicht nur bei der weiblichen erwachsenen Bevölkerung würden die Infektionsraten schnell ansteigen, sondern auch bei den Jugendlichen. Daher würden die Jugendlichen der Stadt einen überdurchschnittlich hohen Anteil an den gesamten Erkrankungsfällen darstellen.

Ohne näher auf mögliche Ursachen für Geschlechtskrankheiten im Allgemeinen oder den Anstieg der Infektionsraten im Besonderen einzugehen, werden von Münx, Rudolf und Bruns prophylaktische Maßnahmen vorgestellt, mit denen in den vergangenen Jahren auf die Verbreitung von Geschlechtskrankheiten im Bezirk und in der Stadt Halle (Saale) reagiert wurde: „Eine wirksame Form der Prophylaxe ist die Durchführung von angeordneten Untersuchungen bei wegen häufig wechselnden Geschlechtsverkehrs und heimlicher Prostitution in Aufsicht stehenden Personen. " Diejenigen Frauen, bei denen diese Form der Prophylaxe nicht wirkte, wurden entweder zur ambulanten Versorgung bestellt oder in eine geschlossene Venerologische Station zwangseingewiesen: „In die Geschlossene Geschlechtskranken-Frauenabteilung des Bezirkskrankenhauses Halle mussten wegen Verstoßes gegen die Geschlechtskrankheiten-Verordnung im Jahre 1977235 Patientinnen kreisärztlich zwangseingewiesen werden. $\mathrm{Zu}$ den Zwangseingewiesenen gehörten nicht nur hallesche Bürgerinnen, sondern auch Bürgerinnen aus dem gesamten Bezirk Halle. " Diese Zwangseinweisungen wurden von Münx und seinen Kollegen damit begründet, dass bei den Frauen der Verdacht auf eine Geschlechtskrank- 
heit vorgelegen habe. Tatsächlich wurde aber im Jahr 1977 nur „bei 30\% der Zwangseingewiesenen eine Geschlechtskrankheit festgestellt und behandelt" ${ }^{15}$ Entsprechend wurde bei etwa 70\% der zwangseingewiesenen Frauen keine Geschlechtskrankheit diagnostiziert. Dennoch wurden auch diese Frauen auf die geschlossene Venerologische Station des Bezirkskrankenhauses Halle (Saale) eingewiesen und zu „vollwertigen Mitgliedern der Gesellschaft“ erzogen, wie es in dem Artikel heißt. ${ }^{16}$

Die zwangsweise Unterbringung krankheitsverdächtiger Personen in geschlossenen Venerologischen Stationen war in der DDR ein etabliertes Instrument zur Vorbeugung und Bekämpfung von Geschlechtskrankheiten, wie aus dem Artikel Zur Bedeutung der Verhütungs- und Bekämpfungsmaßnahmen von $\mathrm{Ge}$ schlechtskrankheiten in der DDR bei „uneinsichtigen Gefährdeten und Kranken“ hervorgeht, abgedruckt in der Dermatologischen Monatsschrift. ${ }^{17}$ Die Autoren Günter Elste, Chefarzt des Städtischen Klinikums Berlin-Buch, und L. Krell legen in diesem Artikel dar, dass 1968 in den 15 Bezirken der DDR insgesamt 2.763 Personen wegen des Verdachts auf eine Geschlechtskrankheit zwangseingewiesen wurden. Differenziert nach Bezirken wurden 1968 in Berlin 508, in Halle (Saale) 436, in Dresden 414, in Leipzig 384, in Rostock 231 sowie in Karl-Marx-Stadt (heute Chemnitz) 164 zwangszugeführte Personen statistisch erfasst. ${ }^{18}$ Bei lediglich 777, also nur 28\% der 2.763 DDR-weit zwangseingewiesenen Personen konnten tatsächlich Erkrankungen wie Syphilis oder Gonorrhoe diagnostiziert werden. Entsprechend lag bei ca. 70\% der Zwangseingewiesenen keine Erkrankung vor. Die zur Unterbringung notwendigen geschlossenen Venerologischen Stationen waren in der DDR über mehrere Bezirke verteilt, wie im „Gertler“, dem dreibändigen Handbuch für Systematische Dermatologie und Grenzgebiete aus dem Jahr 1972/73 nachzulesen ist. ${ }^{19}$ Demnach wurden 1972 in fünf Bezirken der DDR geschlossene Stationen für Geschlechtskranke und Infektionsgefährdete unterhalten. Neben der geschlossenen Station in Halle (Saale) existierte jeweils eine Station in Berlin, Erfurt, Leipzig und Schwerin. ${ }^{20} \mathrm{Im}$ Handbuch von Gertler wurden nicht alle geschlossenen Venerologischen Stationen genannt. So zeigten sich in Unterlagen aus dem Bestand des Bundesarchivs in Berlin (Bestand DQ1: Gesundheitsministerium der DDR), die wir in unserer jüngsten Arbeit zu den geschlossenen Venerologischen Stationen in der DDR ausgewertet haben, dass zumindest in Berlin-Buch, Dresden, Gera, Magdeburg und Rostock ebenfalls geschlossene Stationen für Geschlechtskranke bestanden.

15 Münx, Bruns, Rudolf: Venerische Erkrankungen und ihre Prophylaxe (Anm. 10), S. 70.

16 Münx, Bruns, Rudolf: Venerische Erkrankungen und ihre Prophylaxe (Anm. 10), S. 72.

17 Elste G, Krell L (1970) Zur Bedeutung der Verhütungs- und Bekämpfungsmaßnahmen von Geschlechtskrankheiten in der DDR bei „uneinsichtigen Gefährdeten und Kranken“. Dermatologische Monatsschrift 156, 932-938.

18 Elste, Krell: Zur Bedeutung der Verhütungs- und Bekämpfungsmaßnahmen (Anm. 17), S. 937.

19 Gertler W (1973) Systematische Dermatologie und Grenzgebiete. 3 Bände. Thieme VEB Leipzig.

20 Gertler W (1973) Systematische Dermatologie und Grenzgebiete. Bd. 2. Thieme VEB Leipzig, S. 1363. 
Einigen dieser Stationen widmet der vorliegende Band ein selbständiges Kapitel. ${ }^{21}$

Die unvollständigen Angaben zu den geschlossenen Venerologischen Stationen im Handbuch für Systematische Dermatologie und Grenzgebiete und die Ausführungen von Münx, Rudolf und Bruns werfen eine Reihe von Fragen auf: Gab es für die Zwangseinweisungen eine rechtliche Grundlage? Wie ging eine Zwangseinweisung vonstatten? Welche Funktion hatten diese geschlossenen Stationen, wenn die Frauen dort zu „,vollwertigen Mitgliedern der Gesellschaft" erzogen werden sollten? Wie wurden die Frauen in den geschlossenen Stationen versorgt? Und wie unterschieden sich die einzelnen Einrichtungen in der DDR? Erste Antworten auf diese und weitere Fragen konnten wir im Zusammenhang mit unserer Arbeit zur geschlossenen Venerologischen Station in der Poliklinik Mitte in Halle (Saale) geben. ${ }^{22}$ So ging im Jahr 1961 die geschlossene Venerologische Station am Standort Poliklinik Mitte aus der „Beobachtungs- und Fürsorgestation “ für Geschlechtskranke hervor. Den rechtlichen Hintergrund bildete die im selben Jahr in Kraft getretene Verordnung zur Verhütung und Bekämpfung von Geschlechtskrankheiten vom 23. Februar 1961, ${ }^{23}$ in welcher unter anderem die Zwangseinweisung von Geschlechtskranken in der DDR geregelt war: Personen, die sich ärztlichen Auflagen widersetzten, mehrfach als geschlechtskrank registriert wurden oder unter Verdacht des häufig wechselnden Geschlechtsverkehrs (HwG) standen, sollten stationär behandelt oder in einer geschlossenen Station für Geschlechtskranke untergebracht werden. In der Poliklinik Mitte in Halle (Saale) waren sowohl eine ambulante Station für Haut- und Geschlechtskrankheiten als auch eine geschlossene Venerologische Station eingerichtet. Die geschlossene Station hatte etwa 30 Betten in mehreren Schlafräumen. Sowohl die Eingangstür als auch die Fenster waren vergittert. Neben dieser räumlichen Isolation war jeglicher Besuch durch die Hausordnung untersagt. Mit der Unterbringung auf der geschlossenen Venerologischen Station sollte durch „erzieherische Einwirkung (...) erreicht werden, dass diese Bürger nach ihrer Krankenhausentlassung die Gesetze unseres Staates achten, eine gute Arbeitsdisziplin zeigen und sich in ihrem Verhalten in unserer Gesellschaft von den Prinzipien des sozialistischen Zusammenlebens der Bürger unseres Staates leiten lassen“, wie es in der Hausordnung von 1963 heißt. ${ }^{24}$

Auf die Station wurden Frauen ab dem 12. Lebensjahr gebracht, die wiederholt geschlechtskrank wurden, sich den Auflagen der ambulanten Station (Melde-

21 Steger F, Schochow M (2015) Disciplining through Medicine. Turning Girls and Women into „Socialist Personalities" at Closed Venerological Wards in Communist East Germany (GDR). Droit. Santé et Société. Law Health \& Society [im Druck].

22 Steger, Schochow: Disziplinierung durch Medizin (Anm. 1).

23 Verordnung zur Verhütung und Bekämpfung von Geschlechtskrankheiten vom 23. Februar 1961. Gesetzblatt der Deutschen Demokratischen Republik, Teil II, VEB Deutscher Zentralverlag Berlin 1961, S. 85-89.

24 BArch Best. DQ 1. Nr. 4228, unpag. Hausordnung. Für die Patientinnen der geschlossenen Geschlechtskranken Frauenstation im Stadtkrankenhaus Mitte Halle (S) Kleine Klausstr. 16, Halle (Saale), den 23. April 1963. 
pflicht, Nachkontrolle) widersetzten, im Verdacht standen, eine Geschlechtskrankheit zu haben (meist durch Denunziation) oder als Person mit häufig wechselnden Geschlechtspartnern (HwG-Person) eingestuft wurden. Gerade der Verdacht auf häufig wechselnde Sexualpartner wurde vielfach von Fürsorgestellen oder Ärzten angegeben. Mit dieser Begründung führten Polizisten namentlich bekannte junge Frauen der Station zu, oder die Transportpolizei nahm junge Frauen auf Bahnhöfen willkürlich fest und lieferte sie in die geschlossene Venerologische Station in Halle (Saale) ein. Auf der Station war jede arbeitsfähige Frau zur sogenannten „Arbeitstherapie“ verpflichtet und konnte zu Reinigungsarbeiten der Krankenzimmer bzw. Krankenhausräume aber auch in anderen Objekten der Poliklinik herangezogen werden. Täglich wurden die zwangseingewiesenen Mädchen und Frauen auch gegen ihren Willen gynäkologisch untersucht. In der Regel lag hierfür keine medizinische Indikation vor. Bei der Entnahme des Abstrichs kam es häufig zu Verletzungen, Blutungen und bei Erstabstrichen zu Deflorationen. Die Abstriche wurden auch im Sinn einer abschreckenden und disziplinierenden Maßnahme besonders bei Mädchen und jungen Frauen eingesetzt. Der Behandlungszeitraum betrug vier bis sechs Wochen.

Auf der Station wurde ein hierarchisches Terrorsystem errichtet, das mit der Hausordnung installiert und von den Ärzten, Pflegerinnen und Stubenältesten umgesetzt wurde. Es gab ein System von Belobigungen und Erziehungsmaßnahmen: Belobigungen waren die Erteilung einer zusätzlichen Raucherlaubnis, das Löschen einer Hausstrafe oder schriftliches Lob. Erziehungsmaßnahmen umfassten zusätzliche „Arbeitstherapie“, Rauchverbot sowie die Nachtruhe außerhalb des Bettes auf einem Hocker. Der Leiter der Station handelte unpersönlich und entindividualisierend. Die Pflegerinnen misshandelten die Frauen durch gezieltes Zufügen von Schmerzen während der Abstriche und disziplinierten sie durch Belohnungen (Zuteilung von Zigaretten) und Bestrafungen (Schlafentzug). Dieses Terrorsystem wurde auch durch die Stubenältesten aufrechterhalten und hatte 21 Jahre Bestand. Erst 1982 wurde die Station in Halle (Saale) geschlossen. ${ }^{25}$

Mit der geschlossenen Venerologischen Station in Halle (Saale) wurde an eine Tradition in der medizinischen Versorgung geschlechtskranker Personen angeknüpft, deren Spuren sich bis weit in das 19. Jahrhundert zurückverfolgen lassen..$^{26}$ So zielten seit Mitte des 19. Jahrhunderts staatliche Maßnahmen zur Bekämpfung von Geschlechtskrankheiten auf die Internierung, Festsetzung und Kontrolle Erkrankter in medizinischen Einrichtungen. ${ }^{27}$ Spätestens mit

25 Steger, Schochow: Disziplinierung durch Medizin (Anm. 1).

26 Sauerteig L (1999) Krankheit, Sexualität, Gesellschaft. Geschlechtskrankheiten und Gesundheitspolitik in Deutschland im 19. und frühen 20. Jahrhundert. Steiner Stuttgart.

27 Zschiegner C (2001) Frauen, Schuld, Sühne. Syphilis, Prostitution und Moral von der Mitte des 19. bis in die ersten Jahre des 20. Jahrhunderts. In: Dietrich-Daum E, Matt W, Platzgummer H (Hrsg.) Geschichte und Medizin. Forschungsberichte - Fachgespräche. 241-259. Amt der Stadt Dornbirn, Stadtarchiv Dornbirn. 
der Entdeckung der Erreger von Geschlechtskrankheiten sowie der Entwicklung von Salvarsan als Therapeutikum gegen die Syphilis kam es vermehrt zu Einweisungen von geschlechtskranken Personen in geschlossene Stationen. ${ }^{28}$ Dabei standen die Anwendung und Erprobung des Therapeutikums Salvarsan einerseits sowie die Kontrolle und Regulierung der Prostitution andererseits im Vordergrund. ${ }^{29}$ Wurden in der Weimarer Republik der Gedanke der Prävention $^{30}$ gestärkt und mit dem „Gesetz zur Bekämpfung der Geschlechtskrankheiten "31 vom 18. Februar 1927 die Kompetenzen der Polizei bei der Festsetzung von Prostituierten stark beschnitten, so setzte das „Gesetz gegen gefährliche Gewohnheitsverbrecher und über Maßregeln der Sicherung und Besserung " ${ }_{32}$ vom 24. November 1933 diese Beschränkungen außer Kraft. ${ }^{33}$ Geschlechtskranke Personen, die unter dem Verdacht der Prostitution standen, konnten nun wieder durch Ordnungskräfte in Krankenhäuser zwangseingewiesen werden. ${ }^{34}$

Im Nachkriegsdeutschland wurde an diese Behandlungs- und Verwahrungskonzepte für geschlechtskranke Personen mit geschlossenen Venerologischen Stationen angeknüpft. Sowohl in der Bundesrepublik ${ }^{35}$ als auch in der DDR ${ }^{36}$ wurden entsprechende Stationen eingerichtet bzw. nach Ende des Zweiten Weltkriegs weitergeführt. Während anfänglich die rechtlichen Bestimmungen zur Bekämpfung von Geschlechtskrankheiten in den von den Alliierten besetzten Westzonen Unterschiede aufwiesen, wurden in der Bundesrepublik Deutschland die Zwangsmaßnahmen gegen Geschlechtskranke durch das „Gesetz zur Bekämpfung der Geschlechtskrankheiten“" ${ }^{37}$ vom 23. Juli 1953 ein-

28 Sauerteig L (1996) Salvarsan und der „ärztliche Polizeistaat“. Syphilistherapie im Streit zwischen Ärzten, pharmazeutischer Industrie, Gesundheitsverwaltung und Naturheilverbänden (1910-1927). In: Dinges M (Hrsg.) Medizinkritische Bewegungen im Deutschen Reich (ca. 1870-ca. 1933). 161-200. Steiner Stuttgart.

29 Sauerteig: Salvarsan und der „ärztliche Polizeistaat“ (Anm. 28), S. 180.

30 Gertiser A (2008) Der Schrecken wohnt im Schönen. Darstellung devianter Sexualität in den Aufklärungsfilmen zur Bekämpfung der Geschlechtskrankheiten der 1920er-Jahre. Zeitenblicke 7, urn:nbn:de:0009-9-16249.

31 Gesetz zur Bekämpfung der Geschlechtskrankheiten vom 18. Februar 1927. In: Deutsches Reichsgesetzblatt. Teil I, 1927, S. 536-541.

32 Gesetz gegen gefährliche Gewohnheitsverbrecher und über Maßregeln der Sicherung und Besserung vom 24. November 1933. In: Deutsches Reichsgesetzblatt. Teil I, 1933, S. 995-1008.

33 Pollmeier H (2010) Zwischen Forschung, Therapie und Gesundheitsführung. Die fachöffentliche Diskussion um die Bekämpfung von Geschlechtskrankheiten in Großbritannien und Deutschland (1933-1945). In: Eschenbruch N, Hänel D, Unterkircher A (Hrsg.) Medikale Räume. Zur Interdependenz von Raum, Körper, Krankheit und Gesundheit. 141-175. Transcript Bielefeld, S. 154.

34 Pollmeier: Zwischen Forschung, Therapie und Gesundheitsührung (Anm. 33); Scholz A (2011) „Ein Volk mit Geburtenstillstand oder Geburtenrückgang geht unter". Geschlechtskrankheiten im Nationalsozialismus. In: Krischel M (Hrsg.) Urologen im Nationalsozialismus. Zwischen Anpassung und Vertreibung. 191-202. Hentrich und Hentrich Berlin.

35 Lindner U (2002) Frauen und Geschlechtskrankheiten als Problem der Gesundheitspolitik in Großbritannien und Deutschland. In: Linder U, Niehuss M (Hrsg.) Ärztinnen - Patientinnen. Frauen im deutschen und britischen Gesundheitswesen des 20. Jahrhunderts. 215-241. Böhlau Köln, Weimar, Wien.

36 Brinkschulte E (2011) Bordellbaracken für die Besatzungstruppen. Die Bekämpfung der Geschlechtskrankheiten in Magdeburg in der frühen Nachkriegszeit. In: Puhle M (Hrsg.) Magdeburg lebt! Kriegsende und Neubeginn 1945-1949. 253-268. Magdeburger Museen Magdeburg.

37 Gesetz zur Bekämpfung der Geschlechtskrankheiten vom 23. Juli 1953. In: Bundesgesetzblatt. Teil I. 1953, S. 700-706. 
heitlich geregelt. ${ }^{38}$ Beispiele für geschlossene Stationen in der Bundesrepublik sind vor allem die geschlossenen Abteilungen für Haut- und Geschlechtskrankheiten in den Hafenstädten Bremen und Hamburg, die bereits Gegenstand wissenschaftlicher Untersuchungen waren. So wurden bis Ende der 1940er Jahre in Bremen geschlechtskranke weibliche Prostituierte in die geschlossene Abteilung (Station 5) der Klinik für Haut- und Geschlechtskrankheiten der Städtischen Krankenanstalten eingewiesen. ${ }^{39}$ Nach bisherigem Forschungsstand gab es in Hamburg bis mindestens in die 196oer Jahre eine geschlossene Abteilung für geschlechtskranke Prostituierte. ${ }^{40}$ Für einen umfassenden deutsch-deutschen Vergleich ist weitere Forschung notwendig, insbesondere gilt dies für Städte ohne Häfen.

In der Sowjetischen Besatzungszone (SBZ) und dann in der DDR waren Zwangsmaßnahmen gegen Geschlechtskranke in den frühen Befehlen der Sowjetischen Militäradministration (SMAD) verankert - beispielsweise im SMAD-Befehl Nr. 273, der die „Verordnung zur Bekämpfung der Geschlechtskrankheiten unter der deutschen Bevölkerung in der sowjetischen Besatzungszone Deutschlands vom 11. Dezember 1947" am 1. Januar 1948 in Kraft setzte. ${ }^{41}$ Dieser SMADBefehl Nr. 273 war die rechtliche Grundlage für alle geschlossenen Venerologischen Stationen in der SBZ und in der DDR. Er verlor erst 1961 seine Gültigkeit, als die „Verordnung zur Verhütung und Bekämpfung von Geschlechtskrankheiten" vom 23. Februar 1961 in Kraft trat. ${ }^{42}$ Neben den rechtlichen Grundlagen existierten in der SBZ/DDR auch die institutionellen Voraussetzungen für die zwangsweise Unterbringung von Geschlechtskranken. So wurde beispielsweise 1946 in Leipzig-Thonberg ein geschlossenes Fürsorgeheim für geschlechtskranke Personen eingerichtet, aus dem 1952 eine geschlossene Venerologische Station hervorging. ${ }^{43}$ Fürsorgeheime für Geschlechtskranke existierten auch

38 Scholz A (1999) Geschichte der Dermatologie in Deutschland. Springer Berlin u. a., S. 292.

39 Mörchen S (2011) Schwarzer Markt: Kriminalität, Ordnung und Moral in Bremen 1939-1949. Campus Frankfurt (Main), New York, S. 441; Roelfs A (2006) „Ami-Liebchen“ und „Berufsbräute“. Prostitution, Geschlechtskrankheiten und Besatzungsverhältnisse in der Nachkriegszeit. In: Kronenbitter G (Hrsg.) Besatzung. Funktion und Gestalt militärischer Fremdherrschaft von der Antike bis zum 20. Jahrhundert. 201-209. Schöningh Paderborn, München, Wien, Zürich, S. $205 f$.

40 Foitzik D (1997) „Sittlich verwahrlost“. Disziplinierung und Diskriminierung geschlechtskranker Mädchen in der Nachkriegszeit am Beispiel Hamburg. Neunzehnhundertneunundneunzig 12, 68-82; Reng B, Redhardt R (1968) Prostitution bei weiblichen und männlichen Jugendlichen. Enke Stuttgart, S. 5.

41 Verordnung zur Verhütung und Bekämpfung von Geschlechtskrankheiten unter der deutschen Bevölkerung in der sowjetischen Besatzungszone Deutschlands. SMAD-Befehl Nr. 273 des Obersten Chefs der SMAD vom 11. Dezember 1947. In: Deutsche Zentralverwaltung für das Gesundheitswesen (Hrsg.) Die gesetzlichen Bestimmungen zur Bekämpfung der Geschlechtskrankheiten in der sowjetischen Besatzungszone Deutschlands. S. 15-22. Taschenbuch Verlag Berlin 1948.

42 Verordnung zur Verhütung und Bekämpfung von Geschlechtskrankheiten (Anm. 23); Scholz: Geschichte der Dermatologie in Deutschland (Anm. 38), S. 291.

43 Korzilius S (2004) „Asoziale“ und „Parasiten“ im Recht der SBZ, DDR. Randgruppen im Sozialismus zwischen Repression und Ausgrenzung. Böhlau Köln, Weimar, Wien. 
im Gefängnis im thüringischen Gräfentonna ${ }^{44}$ oder in Brandenburg. ${ }^{45}$ In Berlin wurden nach dem Ende des Zweiten Weltkriegs geschlechtskranke Personen auf eine geschlossene Venerologische Station im Krankenhaus Nordmarkstraße eingewiesen, und in Erfurt gab es seit Mitte der 1950er Jahre eine geschlossene Venerologische Abteilung in der Hautklinik der Medizinischen Akademie. ${ }^{46}$ Mitte der 196oer Jahre hatte sich die Zahl der geschlossenen Stationen für Geschlechtskranke in der DDR bereits vervielfacht.

Im Unterschied zur Bundesrepublik wurden die zwangseingewiesenen Mädchen und Frauen in der SBZ und in der DDR von einer politisierten Medizin versorgt. Diese Medizin war von Akteuren geprägt und betrieben worden, die nicht einfach ein politisches System übernahmen oder sich instrumentalisieren ließen. Vielmehr prägten diese Akteure das politische System selbst durch ihre Normen, die sie bei ihrem täglichen Handeln anwandten. So wurde die medizinische Versorgung stets durch pädagogische Konzepte ergänzt, welche das Ziel hatten, zu „sozialistischen Persönlichkeiten“ zu erziehen. Dieses auf Zwang und Terror aufbauende Disziplinarsystem hatte gravierende Folgen für die zwangseingewiesenen Mädchen und Frauen. Zu den unmittelbaren Folgen gehörten die Defloration bei Erstabstrich (sexualisierte Gewalt), Übelkeit, Durchfall, Erbrechen und Müdigkeit, Kopfschmerzen, langanhaltende Lähmungen, Schüttelfrost und vereinzelt Fehlgeburten auf der Station. Zu den beschriebenen Spätfolgen zählen Angst vor gynäkologischen Untersuchungen und Ärzten, chronische Beschwerden (Schlafstörungen, sexuelle Inappetenz, Inkontinenz), interaktionelle Defizite, Partnerschaftsprobleme, Kinderlosigkeit, gestörte Mutter-Kind-Bindung sowie transgenerationale Weitergabe von (emotionaler) Vernachlässigung und Gewalt. Grundsätzlich leiden die meisten Frauen unter ähnlichen Spätfolgen wie andere Opfer der DDR - beispielsweise die ehemaligen politischen Gefangenen, die von Depressionen und posttraumatischen Belastungsstörungen (PTBS), Angstzuständen oder Albträumen berichten. Wie in Untersuchungen mit politischen Gefangenen der DDR gezeigt werden konnte, leiden viele Befragte immer noch unter den Folgen der Haft. Zwei Fünftel sind von psychischen und Verhaltensstörungen betroffen, klagen vermehrt über chronische Erschöpfung, Schlafstörungen oder Appetitlosigkeit, was unter anderem auf die Verfolgungen und die Haft zurückgeführt wird. ${ }^{47}$

Doch nicht nur politische Haft hat zum Teil lang anhaltende Folgen, wie der Psychologische Psychotherapeut Stefan Trobisch-Lütge von der Berliner Be-

44 Korzilius: „Asoziale“ und „Parasiten“ im Recht der SBZ, DDR (Anm. 43).

45 Pohl D (2001) Justiz in Brandenburg 1945-1955. Gleichschaltung und Anpassung in einer Landesjustiz. Oldenbourg München; Krumbiegel U (2007) Polikliniken in der SBZ/DDR. Konzeption und Umsetzung öffentlicher, poliklinischer Einrichtungen unter der besonderen Berücksichtigung Brandenburgs. VAS Frankfurt am Main.

46 Behling K, Eik J (2007) Vertuschte Verbrechen. Kriminalität in der Stasi. Militzke Leipzig, S. 78.

47 Trobisch-Lütge S, Bomberg KH (Hrsg.) (2015) Verborgene Wunden. Spätfolgen politischer Traumatisierung in der DDR und ihre transgenerationale Weitergabe. Psychosozialverlag Gießen; Beer K, Weißflog G (2011) Weiterleben nach politischer Haft in der DDR. Gesundheitliche und soziale Folgen. Vandenhoeck und Ruprecht Unipress Göttingen. 
ratungsstelle „Gegenwind“ ausführt. ${ }^{48}$ Auch der zwangsweise Aufenthalt in den geschlossenen Stationen für Geschlechtskranke führte zu Traumatisierungen bei den Frauen. Viele fühlen sich heute noch kontrolliert und verfolgt, vertrauen kaum einem Menschen und haben sich teilweise völlig zurückgezogen. Trotz offensichtlicher psychischer Langzeitschäden suchen sich viele Opfer keine Hilfe. Ein Grund dafür sind Scheu, Scham und die verständliche Angst, sich mit den traumatisierenden Ereignissen erneut zu konfrontieren, selbst wenn der Leidensdruck hoch ist. ${ }^{49}$ Für die Opfer der Diktatur der SED (Sozialistische Einheitspartei Deutschlands) ist schon die Auseinandersetzung mit formal-juristischen Anforderungen belastend. Wie wichtig und notwendig spezifische, therapeutische Hilfsangebote sind, zeigen die Opfer, die heute immer noch unter den Folgen des DDR-Regimes leiden. Dies gilt auch für die zwangseingewiesenen Frauen, bei denen zudem zu berücksichtigen ist, dass auch Familienangehörige - beispielsweise Kinder - betroffen sein können und Traumata sich mitunter auf nachfolgende Generationen übertragen. Wie schwierig sich die Verarbeitung des Erlebten gestaltet, zeigte sich auch in den von uns geführten Interviews.

Vor diesem Hintergrund haben wir unsere Forschungsperspektive um zwei Dimensionen erweitert: Zum einen untersuchen wir auf Crundlage unserer Forschungsarbeit zur geschlossenen Venerologischen Station der Poliklinik Mitte in Halle (Saale) die Geschichte der geschlossenen Venerologischen Stationen in der SBZ und der DDR. Dabei gehen wir von Halle (Saale) aus und rekonstruieren die Geschichte ausgewählter Beispiele geschlossener Venerologischer Stationen, die Ende der 1940er Jahre entstanden (Leipzig-Thonberg, Berlin-Buch, Dresden-Friedrichstadt). Es werden auch die Entwicklungen in der SBZ und der frühen DDR berücksichtigt, da in dieser Phase wesentliche rechtliche Grundlagen für die geschlossenen Venerologischen Stationen gelegt wurden und sich Ansätze einer politisierten Medizin entwickelten. Zum anderen gehen wir auf die unmittelbaren Folgen und die Spätfolgen der zwangsweisen Unterbringung in den geschlossenen Venerologischen Stationen ein. Ein wichtiger Aspekt ist dabei die transgenerationale Weitergabe von Traumatisierungen, auf die wir in unseren Publikationen zum Thema bisher nicht explizit eingegangen sind. Für die vorliegende Monographie stehen folgende Fragenkomplexe im Mittelpunkt: 1. Vor welchem institutionellen und rechtlichen Hintergrund wurden die geschlossenen Venerologischen Stationen in der SBZ/DDR eingerichtet? 2. Unterschieden sich die geschlossenen Venerologischen Stationen in der SBZ/DDR in ihrem Aufbau und in ihren Funktionen? 3. Wer arbeitete auf diesen Stationen und welche Mädchen und Frauen wurden dort zwangseingewiesen? 4. Wie wurden die Mädchen und Frauen auf die geschlossenen Venerologischen Stationen eingewiesen? 5. Welche (medizinischen) Eingriffe wurden dort durchgeführt? 6. Wie gestaltete sich der

48 Trobisch-Lütge, Bomberg: Verborgene Wunden (Anm. 47); Trobisch-Lütge S (2004) Das späte Gift. Folgen politischer Traumatisierung in der DDR und ihre Behandlung. Psychosozialverlag Gießen.

49 Steger, Schochow: Disziplinierung durch Medizin (Anm. 1). 
Alltag auf den Stationen? 7. Welchem Terror waren die Mädchen und Frauen in den verschiedenen Stationen ausgesetzt? 8. In welche institutionellen Zusammenhänge waren die geschlossenen Venerologischen Stationen eingebunden? 9. Mit welchen unmittelbaren Folgen und Spätfolgen müssen die Opfer leben, was sind die erlittenen Traumata und werden diese transgenerational weitergegeben?

Unsere bisherigen Ergebnisse zur geschlossenen Venerologischen Station der Poliklinik Mitte in Halle (Saale) wurden auf Grundlage neuer Quellen kritisch geprüft. Beispielsweise wurde aus Akten der Behörde des Bundesbeauftragten für die Unterlagen des Staatssicherheitsdienstes der ehemaligen DDR (BStU) deutlich, dass unsere bisherige Darstellung zur Schließung der Station nur einen Teil der Abläufe darstellt. Inzwischen hat sich gezeigt, dass das Ministerium für Staatssicherheit der DDR sehr früh über die Vorgänge auf der geschlossenen Venerologischen Station in Halle (Saale) informiert war und einen Beitrag an ihrer Schließung hatte. Zudem wurde aus den Unterlagen der BStU klar, dass die geschlossene Venerologische Station der Poliklinik Mitte in Halle (Saale) eine Sonderstellung unter den geschlossenen Venerologischen Stationen in der DDR hatte. So wurde ein ganz erheblicher Teil der Zwangseingewiesenen von Berlin, Leipzig oder Rostock nach Halle (Saale) überwiesen, da unter den verantwortlichen Venerologen die Auffassung vertreten wurde, dass auf dieser Station „Zucht und Ordnung“ herrschte. ${ }^{\circ}$ Vor diesem Hintergrund haben wir unsere Aussagen zur geschlossenen Venerologischen Station der Poliklinik Mitte in Halle (Saale) überarbeitet und stellen im vorliegenden Buch die Ergebnisse in erweiterter Form dar.

Des Weiteren haben wir uns entschlossen, die Namen der beteiligten Ärzte und des Pflegepersonals nicht mehr zu anonymisieren. In unserem Buch Disziplinierung durch Medizin. Die geschlossene Venerologische Station in der Poliklinik Mitte in Halle (Saale) 1961 bis 1982 haben wir eine regionale Geschichte geschrieben und die beteiligten Personen anonym dargestellt, die in der Region bekannt sind. Mit der nun vorliegenden Monographie verlassen wir die regionale und nehmen eine weitere Perspektive ein. Unsere Abkehr von der Anonymisierung begründet sich nicht zuletzt dadurch, dass nach der Publikation des Buches im Jahr 2014 die Namen beteiligter Ärzte und Pflegekräfte in Medienberichten mehrfach öffentlich gemacht worden sind und jederzeit über Mediendatenbanken eingesehen werden können. Allen Zwangseingewiesenen und Zeitzeugen (Ärzten, Pflegerinnen, Fürsorgerinnen, Polizisten oder Bürger), die wir interviewen durften, haben wir die vollständige Anonymität zugesichert. An diese Vereinbarung werden wir uns auch weiterhin halten.

50 BStU Archiv der Außenstelle Halle. MfS BV Halle. KD Halle. VIII 616/76. Treffbericht. Geschlossene Station der Poli-Mitte, Bl. 000095. 